\title{
Factores de riesgo de recurrencia de hernias inguinales
}

\author{
Roger Vega Blanco, Francisco Rodríguez Lara y Marlon Putoy Muñoz \\ Departamento de Emergencia, Hospital Humberto Alvarado Vásquez rogervegablanco@ hotmail.com; \\ Departamento de Pediatría, Hospital Humberto Alvarado Vásquez frankrodrilar@ yahoo.com.mx; \\ Departamento de Cirugía Mixta, Hospital Humberto Alvarado Vásquez, marlonputoy@ hotmail.com
}

Fecha de aprobado: 12 de diciembre de 2015

\section{RESUMEN}

Analizar factores relacionados con recurrencia de hernias Inguinal en Hospital Humberto Alvarado Vásquez. Pacientes y Método: Estudio de Casos y Controles. Periodo Enero 2003 - Diciembre 2010, se efectuaron 805 hernioplastias; 57 casos recurrieron, controles 114 asignados al azar. Criterios de exclusión: menor de 15 años, cirugía de urgencia. El análisis estadístico aplicado, pruebas de Chi cuadrado, ANOVA, y razón de probabilidades (OR) con intervalos de confianza del 95\%. Resultados: Factores asociados a recurrencia hernia: sexo masculino $(\mathrm{p}=0.03)$, mayor de 65 años $(\mathrm{p}=0.007)$, obesidad $(\mathrm{p}=0.0006)$. Según clasificación de Bendavid los tipos de hernias predominantes en casos T1S3D3 y T2S3D3. La técnica quirúrgica más utilizada en casos fue Bassini OR: 2.68 (1.26-5.72; $\mathrm{p}=0.005)$. Se evidenció que las técnicas de Shouldice $(\mathrm{p}=0.007)$ y hernioplastia con malla $(\mathrm{p}=0.0001)$ constituyen un factor protector. Asimismo hubo una relación significativa de casos con uso nylon 2-0 $(\mathrm{p}=000.2)$. El promedio tiempo quirúrgico 50.63 $( \pm 26.8)$ en casos y $41.86( \pm 19.5)$ en controles. La media tiempo de aparición de recurrencia 2 años $( \pm 1.02)$. Conclusiones: Factores de riesgo de recurrencia: masculino, >65 años, obesidad, técnica quirúrgica Bassini y MacVay; factores protectores técnicas de Shouldice y Hernioplastia con malla. Utilizar siempre hilo monofilamento no absorbible.

\section{SUMMARY}

To assess factors associated with recurrent inguinal hernias in Humberto Vasquez Alvarado Hospital. Patients and Methods: Case-Control Study. Period January 2003 - December 2010, 805 hernioplasties were made; 57 cases appealed, 114 randomized controls. Exclusion criteria: under 15 years, emergency surgery. The statistical analysis applied, Chi-square, ANOVA, and odds ratio (OR) with confidence intervals of $95 \%$. Results: Factors associated with recurrence hernia: male gender $(\mathrm{p}=$ $0.03)$, over $65(\mathrm{p}=0.007)$, obesity $(\mathrm{p}=0.0006)$. According Bendavid classification rates prevailing in cases T1S3D3 hernias and T2S3D3. The surgical technique used in most cases was Bassini OR: 2.68 (1.26-5.72; $\mathrm{p}=0.005)$. It was evident that the Shouldice techniques $(\mathrm{p}=0.007)$ and hernia repair mesh $(\mathrm{p}=0.0001)$ constitute a protective factor. There was also a significant association of cases with use nylon $2-0(\mathrm{p}=000.2)$. The average operative time $50.63( \pm 26.8)$ in cases and $41.86( \pm 19.5)$ in controls. The mean time to onset of recurrence 2 years $( \pm$ 1.02). Conclusions: Risk factors for recurrence: Male,> 65 years, obesity, surgical technique and MacVay Bassini; Shouldice hernia repair techniques and mesh protective factors. Always use nonabsorbable monofilament thread. 


\section{INTRODUCCION}

La hernia inguinal ha constituido un tema apasionante para los cirujanos, y objeto de amplio estudio, sin embargo no se ha logrado superar de forma global su histórica complicación: la recurrencia.

Una hernia recurrente aparece en el sitio de la operación inicial con una alteración idéntica a aquella para la cual se llevó a cabo antes la reparación (Castillo, 1999). La tasa de recurrencias aumenta con el número de intentos previos a causa de pérdida de tejidos, friabilidad y retracción cicatrizal;(Flum, Horvath \& Koepsell, 2003; Bisgaard, Bay-Nielsen, Christensen, \& Kehlet, 2007) evidentemente la primera reparación tendrá mayor posibilidad de éxito, y la recidiva es frecuente tras cualquier reparación secundaria de una hernia recurrente. Los factores desencadenantes han sido muy bien estudiados, y uno de los principales es la hiperpresión intraabdominal, originada por tos crónica, prostatismo y estreñimiento; (Lau, Fang, Yuen \& Patil, 2007; Jansen, Klinge, Jansen, \& Junge, 2009; Rosemar, Angerås, Rosengren, \& Nordin, 2010), otros factores asociados constituye un defecto del metabolismo de colágena, debilidad muscular y tensión de la línea de sutura. (Arregui, \& Young, 2005). Antes de la introducción de las técnicas con malla, se aceptaban tasas de recurrencia hasta del 15\% posterior a reparaciones primarias. (Amid, (2005). En México la tasa de recurrencia oscila entre el 3 al 5\% de la población general. ${ }^{1}$ Con el advenimiento de los materiales protésicos, y las técnicas laparoscópicas se han reportado en los mejores centros quirúrgicos cifras de recurrencia en 5 años inferiores a 0.5\%. (Richards, Vipond, \& Earnshaw, 2004; McCormack, Scott, Go, Ross, \& Grant, 2003).

En nuestro país se realiza el esfuerzo de disminuir la recurrencia con el uso de técnicas sin tensión y la aplicación de material protésico; sin embargo, aún no contamos con su implementación en todo el sistema de salud debido causas económicas, carencia de los materiales a emplear, deficiencia en el conocimiento de las técnicas quirúrgicas o por resistencia al cambio de los cirujanos. En Nicaragua no se cuenta con antecedentes de estudios sobre recurrencia de hernias inguinales y sus factores asociados. 


\section{MATERIAL Y MÉTODO}

\section{Población de estudio}

Estudio de casos y controles. Se efectúo en Departamento de Cirugía mixta del Hospital Humberto Alvarado Vásquez de Masaya, Enero 2003 a Diciembre 2010. Es un hospital regional de segundo nivel de atención, cuya área de cirugía cuenta con 42 camas y un equipo de 9 cirujanos generales. En ese periodo 805 pacientes ingresaron con diagnóstico de hernia inguinal, intervenidos quirúrgicamente por tal patología.

\section{Selección de casos}

Lo conformaron 57 pacientes que fueron operados por hernia inguinal y con reaparición del defecto herniario en el mismo sitio donde se realizó la cirugía. Se excluyeron: menores de 15 años y cirugía de urgencia. Dichos casos se identificaron a partir de los registros de estadística del centro. Los controles fueron escogido por método aleatorio simple entre el grupo que no presentó recurrencia, proporción 2 controles por caso.

\section{Recolección de datos}

Se efectúo una búsqueda en el sistema de registro del departamento de Estadística de los egresados del hospital con diagnóstico de cirugía por hernia inguinal, posteriormente se revisaron los expedientes de dichos pacientes intervenidos quirúrgicamente para establecer los casos y controles, y la información se plasmó en una ficha de recolección de datos.

\section{Variables}

| Se estudiaron las siguientes variables: edad, sexo, peso, ocupación, patología asociada, tipo de hernia, tiempo de recurrencia, técnica quirúrgica, material de sutura, tiempo quirúrgico, tiempo estancia intrahospitalaria y complicaciones.

Para determinar el tipo de hernia se utilizó la clasificación de Bendavid, que propone el esquema T.S.D. (tipo, etapa y tamaño en $\mathrm{cm}$ ).

\section{Análisis estadístico}

Se aplicaron pruebas estadísticas de CHI cuadrada (X2), y ANOVA; se utilizó la razón de probabilidades (OR) con intervalo de confianza 95\%. Se empleó el software Epilnfo versión 3.5.2. 


\section{RESULTADOS}

En el período de estudio, 805 pacientes se sometieron a cirugías por hernia inguinal, presentándose 57 recurrencias de la patología (7.08\%). Fueron seleccionados 57 casos y 114 controles. La distribución por género reveló predominio del sexo masculino en casos y controles, $53(92.98 \%)$ y 92(80.71\%); el registro del sexo femenino 4(7.02\%) y 22(19.29\%) respectivamente. Se evidenció mayor riesgo en sexo masculino OR 3.17 (0.96-11.52) X2: estadísticamente significativa (ES) (ver Tabla 1).

La edad media ( $\square$ ) del grupo casos fue 56.12 desviación estándar (DE); 18.28, grupo control $\square$ : 48.67, DE; 19.49. En casos el grupo etáreo con mayor distribución >65 años, 25(43.85\%), seguido por 45-54 años 10(17.54\%); en controles se registró el grupo >65 años 27(23.68\%),55-64 años 22(19.29\%). El riesgo de recurrencia de hernia estuvo asociado con mayores de 65 años OR $2.52(1.21-5.25 ; \mathrm{p}=0.007), \mathrm{X}^{2}$ : ES.

La ocupación más frecuente, grupo de casos: obrero 22(38.6\%), y campesino 15(26.3\%); grupo controles el resultado es similar, 41(36\%) y 33(28.9\%). No se demostró riesgo en ocupaciones obrero OR $1.12(0.55-2.27)$ y campesino OR 0.88 (0.40-1.90) intervalo de confianza no estadísticamente significativa (NES). Se presentó patología médica concomitante en $35(20.4 \%)$. En grupo casos destacan: hipertrofia prostática $14(24.6 \%)$ y obesidad 6(10.5\%); igual en grupo control hipertrofia prostática 6(5.3\%). La recurrencia de hernia inguinal tuvo asociación con obesidad OR: 17.35 (1.96-395.33; p=0.0006), X²: ES, y la hipertrofia prostática, OR 2.48 (0.86-7.47; $\mathrm{p}=0.06) \mathrm{NES}$.

Según el tipo de hernia en grupo casos fueron más frecuentes: T1S3D3 16(28.1\%), T2S3D3 9(15.8\%), T1S2D3 7(12.3\%), T2S2D3 7(12.3\%). La mayor distribución en grupo controles correspondieron a: T1S1D2 38(33.3\%), T2S1D2 23(20.2\%) y T1S1D3 17(14.9). La recurrencia de hernia inguinal tuvo asociación significativa con T1S3D3 OR: $10.73(3.11-40.62 ; p=0.002) \quad y$ T2S3D3 OR: $5.16\left(1.36-21.07 ; \mathrm{p}=0\right.$.) $\mathrm{X}^{2}$ : ES.

En relación a técnica empleada, en grupo casos hubo predominio de: Bassini 23(40.4\%) y McVay 18(31.6\%) pacientes; en grupo de controles destacaron: Shouldice 30(26.3\%) y hernioplastia con malla 29 (25.4\%). Se evidenció la relación con recurrencia de hernia inguinal de Bassini OR 2.68 (1.26-5.72; p= 0.005) X²: ES, y McVay OR 2.04 (0.92-4.53; p= 0.053) X²: NES. 
La técnica de hernioplastia con malla OR $0.05(0-0.38$; $\mathrm{p}=0.0001) \mathrm{X}^{2}$ : ES, y Shouldice OR 0.27 $(0.09-0.79 ; \mathrm{p}=0.007) \mathrm{X}^{2} \mathrm{ES}$ demostraron ser factores protectores.

El material de sutura utilizado frecuentemente en grupo casos correspondió a: Nylon 2-0 29(50.9\%) y Seda 2-0 12(21.1\%). En los controles destacan: Seda 2-0 37(32.5\%) y Nylon 2-0 26(22.8\%). La recurrencia de hernia tuvo asociación con el hilo Nylon 2-0 OR 3.51 (1.68-7.33; p= 0.0002) X²: ES; y el que demostró menor recurrencia fue polipropileno 2-0 con OR 0.19 (0.01$1.47 ; \mathrm{p}=0.07) \mathrm{X}^{2}$ : NES.

La media del tiempo quirúrgico en grupo casos $\square: 50.63$ minutos DE; 26.84, y en grupo controles $\square: 41.86 \mathrm{DE} ; 19.59$, prueba de ANOVA estadísticamente significativa ( $\mathrm{p}=0.02)$. El promedio tiempo de estancia intrahospitalaria en grupos caso y control correspondió a 1.71 día DE; 0.77, y 1.5 día DE;0.55, respectivamente. Se presentaron complicaciones postoperatorias en 15(8.7\%). En las complicaciones inmediatas se obtuvieron en el grupo casos: hematoma 2; en los controles: edema escrotal 1, hematoma 1. Las complicaciones tardías fueron escasas, destacándose en grupo casos infección de herida quirúrgica 5.

El tiempo de aparición de recurrencia de los pacientes intervenidos quirúrgicamente presentó una media $\square: 2$ años DE; 1.02 .

Tabla 1.

\begin{tabular}{|c|c|c|c|c|c|c|}
\hline Variable & & Caso & Control & OR (95\% IC) & $x^{2}$ & $\mathbf{P}$ \\
\hline \multicolumn{7}{|l|}{ Sexo } \\
\hline & Masculino & 53 & 92 & $\begin{array}{c}3.17(0.96- \\
11.52)\end{array}$ & 4.42 & 0.035 \\
\hline & Femenino & 4 & 22 & $\begin{array}{c}0.32(0.09- \\
1.04)\end{array}$ & 4.42 & 0.035 \\
\hline \multicolumn{7}{|l|}{ Edad } \\
\hline & mayor 65 & 25 & 27 & $\begin{array}{c}2.52(1.21- \\
5.25)\end{array}$ & 7.27 & 0.007 \\
\hline & menor de 65 & 32 & 87 & $\begin{array}{c}0.40(0.19- \\
0.83)\end{array}$ & 7.27 & 0.007 \\
\hline \multicolumn{7}{|c|}{ Ocupación } \\
\hline & Campesino & 15 & 33 & $\begin{array}{c}0.88(0.40- \\
1.90)\end{array}$ & 0.13 & 0.718 \\
\hline & Obrero & 22 & 41 & $\begin{array}{c}1.12(0.55- \\
2.27)\end{array}$ & 0.11 & 0.737 \\
\hline
\end{tabular}




\begin{tabular}{|c|c|c|c|c|c|c|}
\hline & $\begin{array}{l}\text { Hipertrofia } \\
\text { prostatica }\end{array}$ & 14 & 6 & $\begin{array}{c}2.48(0.86- \\
7.47)\end{array}$ & 3.43 & 0.064 \\
\hline & Obesidad & 6 & 1 & $\begin{array}{c}17.35(1.96- \\
395.33)\end{array}$ & 11.73 & 0.0006 \\
\hline \multicolumn{7}{|c|}{ Tipo de Hernia } \\
\hline & T1S3D3 & 16 & 4 & $\begin{array}{c}10.73(3.11- \\
40.62)\end{array}$ & 22.07 & 0.002 \\
\hline & T2S3D3 & 9 & 4 & $\begin{array}{c}5.16(1.36- \\
21.07)\end{array}$ & 8.11 & 0.004 \\
\hline \multicolumn{7}{|c|}{ Técnica Quirúrgica } \\
\hline & Bassini & 23 & 23 & $\begin{array}{c}2.68(1.26- \\
5.72)\end{array}$ & 7.82 & 0.005 \\
\hline & McVay & 18 & 21 & $\begin{array}{c}2.04(0.92- \\
4.53)\end{array}$ & 3.72 & 0.053 \\
\hline & Shouldice & 5 & 30 & $\begin{array}{c}0.27(0.09- \\
0.79)\end{array}$ & 7.14 & 0.007 \\
\hline & $\begin{array}{l}\text { Hernioplastia } \\
\text { con malla }\end{array}$ & 1 & 29 & $\begin{array}{c}0.05(0.00- \\
0.38)\end{array}$ & 14.65 & 0.0001 \\
\hline \multicolumn{7}{|c|}{ Material de sutura } \\
\hline & Nylon 2-0 & 29 & 26 & $\begin{array}{c}3.51(1.68- \\
7.33)\end{array}$ & 13.64 & 0.0002 \\
\hline & Seda $2-0$ & 12 & 37 & $\begin{array}{c}0.55(0.24- \\
1.24)\end{array}$ & 2.4 & 0.121 \\
\hline & $\begin{array}{l}\text { Polipropileno } \\
2-0\end{array}$ & 1 & 10 & $\begin{array}{c}0.19 \text { (0.01 - } \\
1.47)\end{array}$ & 3.09 & 0.078 \\
\hline \multicolumn{7}{|c|}{ Tiempo quirúrgico } \\
\hline & $\begin{array}{l}\text { Promedio } \\
\text { (minuto) }\end{array}$ & $\begin{array}{c}50.63 \\
( \pm 26.8) \\
\end{array}$ & $\begin{array}{c}41.86 \\
( \pm 19.5) \\
\end{array}$ & & & \\
\hline \multicolumn{7}{|c|}{ Tiempo aparición recurrencia } \\
\hline & $\begin{array}{l}\text { Promedio } \\
\text { (año) }\end{array}$ & $\begin{array}{c}2 \\
( \pm 1.02)\end{array}$ & & & & \\
\hline
\end{tabular}

\section{DISCUSIÓN}

La tasa recurrencia de hernia inguinal oscila entre 0.2 y $10 \%$, hecho que justifica su interés de estudio a nivel mundial. (Barrat, Surlin, Bordea, \& Champault, 2003; Sevonius, Gunnarsson, Nordin, Nilsson, \& Sandblom, 2009;). La incidencia de recurrencia fue $7.08 \%$ en el periodo de estudio, similar a cifras reportadas en contextos de acceso limitado a materiales protésicos y nuevas técnicas endoscópicas. (McCormack, et. al., 2003; Purkayastha, Chow, Athanasiou, Tekkis, \& Darzi, 2008; Cheek, Black, Devlin, Kingsnorth, Taylor, \& Watkin, 1997). En general las hernias son 5 veces más frecuentes en hombres, y el estudio muestra que el riesgo de recurrencia se triplicó en pacientes del sexo masculino $(\mathrm{p}=0.03)$. 
La ingle es una de las áreas débiles naturales de la pared abdominal, y se le atribuye un debilitamiento muscular posterior a los 50 años. (Schwartz, 1995). Los pacientes mayores de 65 años tuvieron el mayor riesgo de recurrencia $(\mathrm{p}=0.007)$. Pueden existir diversos factores predisponentes congénitos o constitucionales, donde cobra gran importancia como desencadenante la hiperpresión abdominal que se relaciona con ciertas ocupaciones y patologías; (Lau, et. al. 2007; Rosemar, et. al., 2010) en el estudio predominó el grupo de obreros y campesinos, aunque no demostraron riesgo significativo de recurrencia ( $\mathrm{p}>0.05)$. La obesidad incrementó 17 veces el riesgo de recurrencia $(\mathrm{p}=0.006)$, hipertrofia prostática fue comorbilidad más frecuente pero su asociación con recurrencia es NES $(\mathrm{p}=0.06)$. Debe enfocarse la reducción de peso en pacientes obesos previo a cirugía de reparación herniaria.

Según tipo de hernias, predominó en recurrencias el T1S3D3 y T2S3D3, hecho fundamentado en que las hernias de mayor tamaño ocasionan destrucción marcada de la pared posterior y tienden a recurrir con mayor frecuencia que las de pequeño tamaño.(Jansen,et. al., 2009; Kingsnorth, \& LeBlanc, 2013). Con el advenimiento de técnicas laparoscópicas y el uso de materiales protésicos se han logrado en centros quirúrgicos especializados tasas de recurrencias inferiores al 1\%, ocasionando disminución en el empleo de técnicas como Bassini y McVay las cuales presentan tasas de recurrencia de 5-20\% y 3-6\% respectivamente, por lo que su uso debe limitarse. (Amid, (2005; Slater, Hopkins, \& Bailey, 2001). En el estudio se duplicó el riesgo de recurrencia con la técnica de Bassini $(\mathrm{p}=0.005)$, y McVay $(\mathrm{p}=0.053)$ no demostró asociación estadística significativa. Técnica de Shouldice y Lichtenstein (Amato, et. al., 2009; Nienhuijs, Van Oort, Keemers-Gels, Strobbe, \& Rosman, 2005) se reportan con recurrencia de 0.6-2.6\% y menor a $1 \%$ en series internacionales; el estudio evidenció el empleo de ambas técnicas como factores protectores, hernioplastia con malla $(\mathrm{p}=0.0001)$, y Shouldice $(\mathrm{p}=0.007)$.

El uso de los distintos materiales en la reparación de la hernia inguinal ha interesado a los cirujanos hace varias décadas; una reparación bajo tensión y el uso de material absorbible. (Jansen,et. al., 2009; Vrijland, et. al., 2002) son errores técnicos que deben ser evitados. Se evidenció que el hilo nylon 2-0 aumentó 3.5 veces el riesgo de recurrencia ( $\mathrm{p}=0.0002)$. Los materiales de sutura no absorbibles, de monofilamento, son los que presentan mayores ventajas para evitar recurrencias. (Lichtenstein, Shulman, \& Amid, 1993). El uso de polipropileno 2-0 no demostró efecto protector $(\mathrm{p}=0.07)$. 
Los pacientes que presentaron recidiva tuvieron un tiempo quirúrgico prolongado en comparación con el grupo control, prueba ANOVA ES. Algunos autores han destacado que la prolongación del acto quirúrgico eleva el riesgo de infección de la herida operatoria cuando supera los 60 minutos; (Mangram, et. al.1999; Taylor, et. al., 2004) así mismo, las hernias recurren con más frecuencia cuando hay infección postoperatoria de la herida. En el grupo de casos hubo 5 pacientes con infección de herida, y fueron pocas otras complicaciones (inmediatas y tardías): hematoma, edema escrotal, dolor y parestesia.

La recurrencia temprana ocurre durante los dos primeros años posteriores a la operación inicial y se debe a inadecuada tensión sobre la línea de sutura o error en la técnica quirúrgica, en cambio la recurrencia tardía sucede después de 2 años y resulta del debilitamiento de la pared posterior y metabolismo anormal de colágena. ;(Flum,et. al., 2003; Bisgaard, et. al., 2007). El estudio demostró una media del tiempo de recurrencia de 2 años DE (1.02), influido por factores mencionados previamente. Los pacientes operados por hernia inguinal deben tener un seguimiento mínimo de 2 años.

Innumerables avances han revolucionado el campo de las hernias inguinales, y dado su complejidad y alto interés se siguen dedicando esfuerzos e innovaciones en la técnica para curar las hernias, para evitar la recurrencia y tratar las mismas.

\section{REFERENCIAS}

Amato, B., Moja, L., Panico, S., Persico, G., Rispoli, C., Rocco, N., \& Moschetti, I. (2009). Shouldice technique versus other open techniques for inguinal hernia repair. Cochrane Database Syst Rev, 4.

Amid, P. K. (2005). Groin hernia repair: open techniques. World journal of surgery, 29(8), 10461051.

Arregui, M. E., \& Young, S. B. (2005). Groin hernia repair by laparoscopic techniques: current status and controversies. World journal of surgery, 29(8), 1052-1057.

Barrat, C., Surlin, V., Bordea, A., \& Champault, G. (2003). Management of recurrent inguinal hernias: a prospective study of 163 cases. Hernia, 7(3), 125-129. 
Bisgaard, T., Bay-Nielsen, M., Christensen, I. J., \& Kehlet, H. (2007). Risk of recurrence 5 years or more after primary Lichtenstein mesh and sutured inguinal hernia repair. British journal of surgery, 94(8), 1038-1040.

Castillo A, Perea A y cols. (1999). Consenso. Hernia Inguinal. Asoc Méx Cir Gral

Cheek, C. M., Black, N. A., Devlin, H. B., Kingsnorth, A. N., Taylor, R. S., \& Watkin, D. F. (1997). Groin hernia surgery: a systematic review. Annals of the Royal College of Surgeons of England, 80, S1-80.

Flum D.R, Horvath K. \& Koepsell T. (2003). Have outcomes of incisional hernia repair improved with time? A population-based analysis. Ann Surg 237(1), 129-135.

Jansen, P. L., Klinge, U., Jansen, M., \& Junge, K. (2009). Risk factors for early recurrence after inguinal hernia repair. BMC Surgery, 9(1), 18.

Lau H., Fang C., Yuen W.K. \& Patil, N.G. (2007). Risk factors for inguinal hernia in adult males: a case-control study. Surgery. 141(2), 262-6.

Lichtenstein, I. L., Shulman, A. G., \& Amid, P. K. (1993). The cause, prevention, and treatment of recurrent groin hernia. The Surgical clinics of North America, 73(3), 529-544.

McCormack, K., Scott, N., Go, P. M., Ross, S. J., \& Grant, A. (2003). Laparoscopic techniques versus open techniques for inguinal hernia repair. The Cochrane Library.

Purkayastha, M. S., Chow, M. A., Athanasiou, M. T., Tekkis, P. P., \& Darzi, A. (2008). Inguinal hernia. Clinical evidence, 2008.

Richards, S. K., Vipond, M. N., \& Earnshaw, J. J. (2004). Review of the management of recurrent inguinal hernia. Hernia, 8(2), 144-148.

Rosemar, A., Angerås, U., Rosengren, A., \& Nordin, P. (2010). Effect of body mass index on groin hernia surgery. Annals of surgery, 252(2), 397-401.

Schwartz, S. y Col. (1995). Principios de cirugía. (6ta. ed.) Interamericana. McGraw-Hill. Vol. 2.

Sevonius, D., Gunnarsson, U., Nordin, P., Nilsson, E., \& Sandblom, G. (2009). Repeated groin hernia recurrences. Annals of surgery, 249(3), 516-518. 
Slater, G. H., Hopkins, G., \& Bailey, M. (2001). Mesh compared with non-mesh methods of open groin hernia repair: systematic review of randomized controlled trials (Br J Surg 2000; 87: 854-9) and Laparoscopic compared with open methods of groin hernia repair: systematic review of randomized controlled trials (Br J Surg 2000; 87: 860-7). British Journal of Surgery, 88(3), 470-471.

Vrijland, W. W., van Den Tol, M. P., Luijendijk, R. W., Hop, W. C. J., Busschbach, J. J. V., de Lange, D. C. D., ... \& Jeekel, J. (2002). Randomized clinical trial of non-mesh versus mesh repair of primary inguinal hernia. British journal of surgery, 89(3), 293-297. 